\title{
Memristor, a Nano-Scaled Element for the Computer Memory: A Mini-Review with Some New Results for an ac- Driven Memristor
}

\author{
Elena Zhitlukhina ${ }^{1}$, Mikhail Belogolovskii ${ }^{2}$ \\ ${ }^{1}$ Dept. of Dynamical Properties of Complex Systems, Donetsk Institute for Physics and Engineering, National Academy of Sciences of \\ Ukraine, Kyiv, Ukraine \\ ${ }^{2}$ Lab. of Dynamics of Electronic Processes in Hybrid Structures, Institute for Metal Physics, National Academy of Sciences of Ukraine, Kyiv, \\ Ukraine
}

\section{Email address:}

elena_zhitlukhina@ukr.net (E. Zhitlukhina),belogolovskii@ukr.net (M. Belogolovskii)

\section{To cite this article:}

Elena Zhitlukhina, Mikhail Belogolovskii. Memristor, a Nano-Scaled Element for the Computer Memory: A Mini-Review with Some New Results for an ac-Driven Memristor. Journal of Photonic Materials and Technology. Vol. 1, No. 2, 2015, pp. 27-32. doi: $10.11648 /$ j.jmpt.20150102.12

\begin{abstract}
In this paper, we give a short look at the concept of memristive nano-technology, its history, and actual state-ofthe-art. It is expected that together with advanced light-driven data transfer technology, computer operation will change dramatically by memristors, a new kind of the computer memory, which is becoming a sustaining hotspot in fields of physics and electronics. This entry aims to familiarize scientists working in the field of photonics with the phenomenon of resistive switching in ac-driven memristors. In addition to already published results, we present our original interpretation of resistance changes in heterostructures based on complex oxides with oxygen vacancies as the most moveable component under applied electric fields.
\end{abstract}

Keywords: Electric Fields, Multilayered Structures, Resistive Switching, Memristive Technology, Complex Oxides, Oxygen Vacancies

\section{Introduction}

Last year, the scientific community learned about the development of a new form of computer architecture that brings together advancements in nano-scale manufacturing and fiber optics. With this new approach, using electrons for computation, photons for communication, and ions for storage, researchers at Hewlett-Packard are going to change dramatically the way how computers are operating today [1]. Whereas the first issue is well developed at the moment, the company is working on applying the high-speed potential of fiber optics inside computers, in place of traditional copper wires. In parallel with its light-driven data transfer technology (high-speed silicon photonic interconnects), researchers are working on a new kind of the computer memory called memristor, a nanoscale chip which increases both the storage capacity and speed of memory. And it is just the third part of the ambitious project. A network of memristors can be realized using a grid of wires with layers of extremely thin nano-ionic materials stacked up at each intersection. As a result, the novel computers with unprecedented amounts of memory will be able to store huge amounts of information.

It should be noted that, comparing to two other new components of the computer architecture, memristive nanotechnology as well as its physical principles are less known. The short overview offers an introductory background on memristors, the history, and the main concepts of the memristive behavior when it is caused by movement of ions. In addition to the already published results, we present our original interpretation of resistance changes in complex oxides with oxygen vacancies as the most moveable component under electric fields applied. The main aim of the overview is to familiarize a reader from the photonics community to this new exciting field of nano-ionics.

\section{The Main Concept}

In 1971 Prof. L.O. Chua introduced a theoretical concept of what he defined as the fourth fundamental passive device named as memory-resistor (memristor for short) [2]. 
According to the paper [2], operation of conventional electronic linear circuits is described by four instantaneous values of fundamental variables, the voltage $v$, the current $i$, the charge $q$, and the magnetic flux $\varphi$ (time integration of voltage). At the same time, there are only three independent two-terminal passive circuit parameters, the resistance $\mathrm{R}$, the capacitance C, and the inductance L. Prof. L.O. Chua argued that there should be a fourth fundamental circuit element (memristor) which connects a missing link (Fig. 1, dashed line) between charge and flux completing the functional relationships between the four fundamental circuit variables. $\mathrm{He}$ found that this new nonlinear circuit element is essentially a resistor with memory, namely, its resistance depends on the amount of charge that flowed through the device. It is important that this conclusion does not depend on any physical mechanism that might couple the flux and charge and did not even require causality. Memristance $M$ in Fig. 1 is identical to resistance only in the linear case, but if $M$ is itself a function of charge, it is independent: A nonlinear, passive memristic device cannot be made with networks of $L, C$, and $R$ without active components (batteries), and this is the significance of having a memristor $M[2]$.

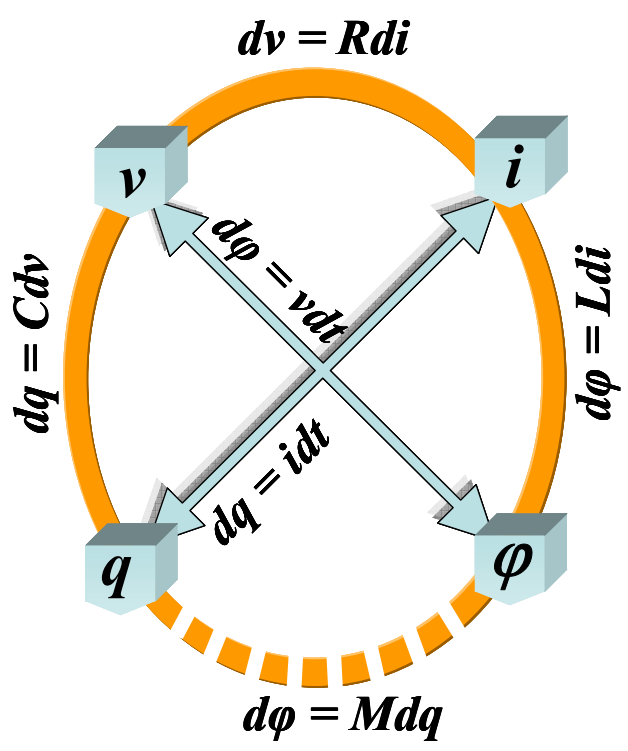

Figure 1. Four instantaneous values of fundamental variables of an electrical circuit, voltage $v$, current $i$, electrical charge $q$, and magnetic flux $\varphi$. Functional relationships between them are defined by basic parameters, the resistance $R$, the capacitance $C$, the inductance $L$, and the memristance $M$. The missing link between charge and flux is shown by the dashed line.

The memristor, a two-terminal passive device may be either current- or voltage-driven. As was argued above, the resistance of a memristor depends on the integral of the input applied to the terminals (rather than on the instantaneous value of the input as in a varistor), i.e., is uniquely defined by the past values of current through it or voltage across it depending on the controlling source. After the current (or voltage) is turned off, the memristor keeps its conductivity level under no driving condition, effectively providing the way to remember basic electrical information. If a memristor is driven with some type of cyclic excitation, such as a sinusoidal current, the plot of the voltage vs. the current will be a Lissajous curve for which the voltage is always equal to zero when the current is zero, and vice versa. Such hysteretic behavior is the main specific feature characterizing memristors as a specific class of dynamical systems. Different from the conventional dielectric breakdown effect which leads to a permanent resistance decrease so that switching back is impossible, the resistance-switching process is reversible and can be repeated a certain (usually, very large) number of times.

In general, there are two types of resistance switchings, unipolar and bipolar [3]. In the first case, switching depends only on the magnitude of the applied bias and is usually related to Joule heating inside the device [3]. In the following, we limit ourselves to bipolar ones schematically shown in the main panel of Fig. 2. In bipolar switching, the memristive device changes its resistance depending on the polarity and magnitude of the applied driving source. For example, the device starts in on- (low-resistance) state and can be transformed into off- (high-resistance) state when a positive voltage larger than threshold voltage $\mathrm{V}_{+}$is applied to the top electrode, while a negative voltage larger than another threshold voltage $\mathrm{V}_{\text {. }}$ transforms the device back to on- (lowresistance) state. What is important is that the device resistance state is not affected significantly if the applied bias is between the two threshold voltages $\mathrm{V}_{+}$and $\mathrm{V}_{\text {-, }}$, enabling the low-voltage read process. Since the bipolar switching is dependent on the polarity of applied bias it is usually associated with the electric field inside the device.

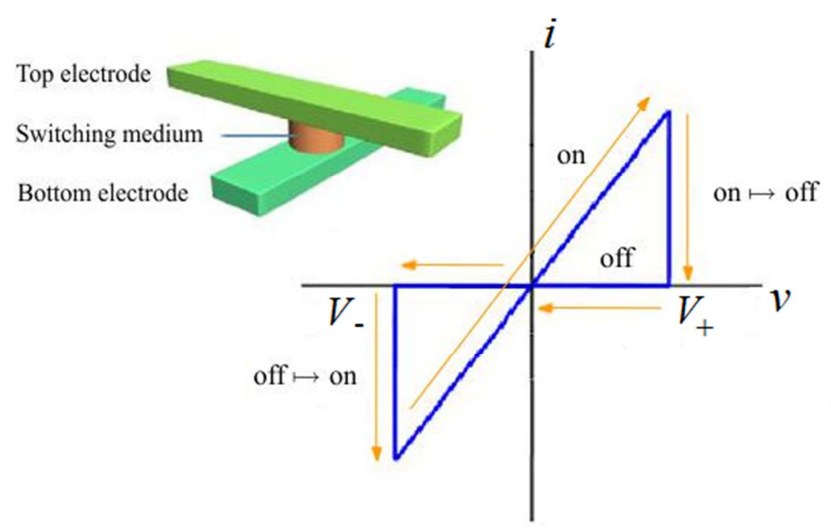

Figure 2. Bipolar resistance-switching $i$-v characteristic. The inset shows a typical metal-switching medium-metal trilayer configuration which exhibits the hysteretic behavior.

\section{Resistive Switching Phenomenon in Transition-Metal Oxides}

Switching medium in the conventional sandwich configuration shown in the inset in Fig. 2 is usually an oxide insulator. First studies of the electric-field-induced resistance switching phenomenon in such materials were comprehensively reviewed already forty five years ago by Dearnaley et al [4]. But the lack of progress in interpreting 
and controlling the effect significantly reduced the related activities. The renewed interest in this phenomenon was caused by the discovery of the high-temperature superconductivity in cuprates and the colossal magnetoresistance effect in manganites following by extended experiments on perovskites which provided new knowledge about their electrical properties. In the early $90 \mathrm{~s}$ the low-resistive switching effect was observed in the point contacts formed by a silver electrode and single-crystal yttrium- and hafnium-based cuprates [5]. At temperature 4.2 $\mathrm{K}$, the authors found reversible polar-dependent switching current-voltage characteristics between states with different excess currents and linked this phenomenon with oxygen electromigration in the crystal lattice of superconducting cuprates. Further investigations [6] have confirmed the presence of the effect in a high- $T_{\mathrm{c}}$ superconductor $\mathrm{BiSrCaCuO}$ and showed that it is observed up to room temperature. Experiments performed on four-component Mnbased compounds revealed the presence of resistive switchings in this class of materials as well $[7,8]$.

At the same time it became clear that this effect can provide a new application for non-volatile memory called resistance random-access memory (RRAM or ReRAM) [3] which works by changing the resistance across an oxide interlayer between metallic electrodes as it is shown in the inset in Fig. 2 and is promising for its easy fabrication, fast switching speed $(<10 \mathrm{~ns})$, high scalability, and good compatibility with the conventional Si-based complementary metal-oxide semiconductor technology. Moreover, sometimes it is able to switch the trilayer to several resistance states. It means that the storage density of one memory cell can be multiplied without changing the cell volume and thus the ReRAM has the potential to realize a multilevel memory. ReRAM is recently under development by a number of companies. Different forms of the cells have been disclosed, based on large variety of materials, spanning from perovskites and more simple binary transition-metal oxides to carbon-based materials and porous silicon-oxide dielectric. Elementary ReRAM cells can be put together within crosspoint architecture that may enable vertically stacking memory layers, ideally suited for mass-storage devices.

In 2008, Strukov et al at HP Labs [9] have successfully identified the trilayers shown n Fig. 2 as a memristive system and subsequently proposed a broader range of applications including neuromorphic systems. In their device a very thin $(\sim 5 \mathrm{~nm}) \mathrm{TiO}_{2}$ film is sandwiched between two nanometerthick metallic electrodes. In the initial state, one side of the $\mathrm{TiO}_{2}$ was doped with oxygen vacancies, which are positively charged. Such doping lowers the resistance of the film to electrons and thus results in two different regions: a highresistance (undoped) and a low-resistance (doped) ones. Fig. $3 \mathrm{a}$, left shows the spatial variation of the doping level in an initially inhomogeneous titanium dioxide. When an electric field is applied, the oxygen vacancies drift moving the boundary between high-resistance and low-resistance regions. Thus the total resistance of the oxide interlayer is dependent on how much charge, an integral of the input current applied to the terminals, has been passed through it in a particular direction. As the width of the doped region grows, the overall resistance decreases. When the $\mathrm{TiO}_{2}$ layer is totally depleted, it is equivalent to the low-resistance state (Fig. 3b, left). The inverse situation corresponds to the high-resistance state (Fig. $3 \mathrm{c}$, left).

The original proposal of a memristor envisioned an alternate-current (ac) device. When a sinusoidal driving voltage is applied, the charge-dependent change in the device resistance results in a frequency-dependent Lissajous-figure current-voltage characteristic [9] whose simulation for a particular case of a switching cuprate-based medium is shown in Fig. 3, right (see below). This dynamical effect arises due to the fact that the memristor response to a periodic perturbation includes contributions with frequencies that are multiples of the initial one. That is why the measured $I-V$ curve appears to be double-valued like Lissajous figures that arise when a point is moving in two perpendicular directions with different frequencies. Note that the simulation does reproduce the main features typical for bipolar switching.
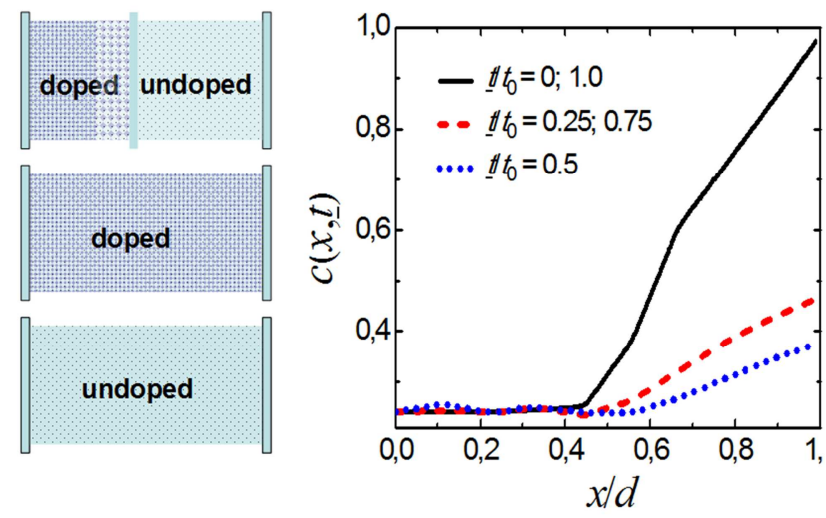

Figure 3. Spatial distribution of oxygen vacancies. Left: schematic of the distribution of oxygen vacancies in a non-uniform titanium-dioxide interlayer located between two metal electrodes in initial, low-resistance, and high-resistance states (from top to bottom). Right: simulation of the time-dependent distribution of oxygen vacancies in an ac-driven metal$Y B C O$ cuprate contact using Eq. (3) with parameters $\beta=0.07$ and $\gamma=10^{5}$, $t_{0}$ is the period of the alternating current bias, $d$ is the thickness of the YBCP layer.

\section{Physical Mechanisms of Resistive Switching in Transition-Metal Oxides}

At the same time, we should notice that the resistance switching phenomenon is observed in a broad range of materials and the exact switching mechanism can significantly differ in diverse materials. There are two basic categories of the models used to describe the underlying physics, those of an electronic origin like Mott metalinsulator transition [10] or carrier trapping/detrapping [11,12] and others of an ionic nature such as cation- and anionmigration [13,14], interfacial Schottky barrier narrowing [15], etc. Independently on which mechanism governs the switching behavior, almost all models agree in opinion that 
the resistance switching phenomenon in oxides occurs only in a small confined region whether near the metal/oxide interface, or inside the bulk of ultra-thin oxides accompanied by the formation and rupture of conductive channels.

In case of unipolar devices, switching only depends on the magnitude of the applied bias. It is naturally to assume that unipolar switching involves Joule heating inside the sample $[16,17]$. In bipolar-switching devices which will be discussed below the effect is dependent on the polarity of the applied bias and thus the switching is usually associated with electric field inside the oxide. The two most popular models are metal ion injection from the electrodes [18] and drift of oxygen vacancies [19] which was been already discussed above.

To provide some arguments in support of any particular theory we should know as more as possible about inhomogeneous states in the switching media. To do it, we need a model material which has been already studied in detail. For this purpose, we use an yttrium-based cuprate $\mathrm{YBa}_{2} \mathrm{Cu}_{3} \mathrm{O}_{7-c}$ (YBCO), a high-temperature superconductor famous by its critical temperature about $90 \mathrm{~K}$. Whereas $\mathrm{YBa}_{2} \mathrm{Cu}_{3} \mathrm{O}_{7}$ is a well-defined chemical compound with a specific structure and stoichiometry, cuprates with fewer than seven oxygen atoms per formula unit are non-stoichiometric. The structure and physical characteristics of these materials depend on the oxygen vacancies content. This nonstoichiometry is denoted by the $c$ in the chemical formula $\mathrm{YBa}_{2} \mathrm{Cu}_{3} \mathrm{O}_{7-c}$.

In our opinion, just experiments with YBCO films can provide important information concerning the nature of the phenomenon in transition-metal oxides due to three reasons. First, this material is a layered compound with stacks of conducting $\mathrm{CuO}_{2}(a-b)$ planes and hopping charge transport along the normal $c$ axis. Because of it, oxygen migration in $\mathrm{YBCO}$ is highly anisotropic with diffusion in the $a-b$ plane much faster than that along the $c$ axis [20] and it can be used to control the oxygen kinetics. Second, at low temperatures the compound is superconducting which opens new possibilities for monitoring the state of the metal-oxide interface. Third, there is an approximate one-to-one relation between the homogeneous $c$-axisoriented YBCO film resistivity $\rho$ and oxygen stoichiometry [21] $\rho(c)=\rho_{0} f(c)$ with $f(c)=\exp (5 c)$ which allows quantitative modeling of the effect.

In contrast to the experimental study [21] we are dealing with spatially and temporarily dependent concentration $\mathrm{c}(\mathrm{x}, \mathrm{t})$, $\mathrm{x}$ is the normal to the oxide surface along the c-axis, all characteristics are assumed constant over the a-b plane. Following experiment [22] we consider a bilayer metal - caxis cuprate configuration instead of a conventional trilayer and assume that the driving factor is an external alternating current $\mathrm{I}(\mathrm{t})=\mathrm{I}_{0} \sin \Omega \mathrm{t}$ passed through the bilayer. Estimation of the vacancy concentration $\mathrm{c}(\mathrm{x}, \mathrm{t})$ versus time and distance makes it possible to calculate the total YBCO film resistance
$R(t)=\int_{0}^{d} \rho(x, t) d x$ where $\rho(x, t)=\rho_{0} \exp (5 c(x, t)), d$ is the oxide film thickness. The key relations describing the oxygen subsystem are (i) the conventional mass balance (continuity) equation for the concentration of vacancies, which includes the total vacancy flux $J(x, t)$ and (ii) the equation for $J(x, t)$ which is a sum of two fluxes caused by different ionmigration forces. The diffusion flux is related to a concentration gradient and equals to $J_{\text {dif }}(x, t)=-D \partial c(x, t) / \partial x$ where $D$ is the vacancy diffusion constant. Besides it, there is a direct effect of the local external electric field $\mathrm{E}(\mathrm{x}, \mathrm{t})$ acting on a vacancy (ion). In the linear-response approximation the drift flux equals to the product $J_{\text {drift }}(x, t)=\frac{\operatorname{Dqc}(x, t)}{k_{B} T} E(x, t)$ where $q$ is the ion charge, $\mathrm{k}_{\mathrm{B}}$ is the Boltzmann's constant, $\mathrm{T}$ is the temperature, the Einstein relation between the charge mobility and diffusivity was used, the electric field $E(x, t)=\rho(x, t) I(t)$, $\rho(x, t)$ is the local oxide-film resistance per unit length which is determined by the local concentration $c(x, t)$ of oxygen vacancies. Next, we assume that this dependence has the same empirical form as for oriented along the axis c homogeneous bulk layers with different oxygen content. As a result, the total flux

$$
J(x, t)=-D \frac{\partial c(x, t)}{\partial x}+\frac{\operatorname{Dqc}(x, t)}{k_{B} T} \rho(x, t) I(t) .
$$

Current flow through the oxide layer with mobile vacancies can imply their spatially dependent profile and, if it changes in time, the $\mathrm{c}(\mathrm{x}, \mathrm{t})$ function should be determined in a time domain. The non-linear behavior of the local electric field acting on the vacancies leads to a double-valued dependence of the current through the metal-cuprate contact on the voltage bias.

The continuity equation is a consequence of the conservation of the number of vacancies in the sample

$$
\frac{\partial \mathrm{c}(\mathrm{x}, \mathrm{t})}{\partial \mathrm{t}}=-\frac{\partial \mathrm{J}(\mathrm{x}, \mathrm{t})}{\partial \mathrm{x}}+\mathrm{S}(\mathrm{x}, \mathrm{t})
$$

where $\mathrm{S}>0 \quad(\mathrm{~S}<0)$ describes the source (the drain) of vacancies. Within a homogeneous sample at equilibrium $\mathrm{S} \equiv 0$ but unbalanced vacancy subsystem will seek to return to the initial state $\mathrm{c}_{\text {in }}(\mathrm{x})$ and $\mathrm{S}(\mathrm{x}, \mathrm{t}) \simeq-\left(\mathrm{c}(\mathrm{x}, \mathrm{t})-\mathrm{c}_{\text {in }}(\mathrm{x})\right) / \tau$, here $\tau$ is the relaxation time. At the temperature $T \sim 100 \mathrm{~K}$ of the experiment [22] this process in the oxide bulk will go very slowly as the probability of formation of new (or disappearance of existing) vacancies is close to zero. However, at the film surface vacancies are able to overcome a relatively low potential barrier, and the effect can be appreciable. Specific value of $S(x, t)$ will be determined by the environment, the gas pressure and the oxygen saturation.

The system of the equations (1) and (2) contains several fitting parameters. To verify the hypothesis that in the case of cuprates the controlling factor is the electric field effect on 
the oxygen-vacancies subsystem, it is necessary to compare results of the simulations with intentionally prepared YBCO samples where the charge transport is almost onedimensional and occurs just in the c-axis direction. The latter condition was ensured in [22] by an additional layer of $\mathrm{SiO}_{2}$ through which direct contact of $\mathrm{Ag}$ and c-axis $\mathrm{YBCO}$ film was carried out exclusively in the direction normal to the oxide surface.

Numerical calculations of the current-voltage curves were performed as follows. By combining the equations (1) and (2), we obtain the nonlinear partial differential equation for the distribution of vacancies $\mathrm{c}(\mathrm{x}, \mathrm{t})$

$$
\begin{aligned}
& \frac{\partial c(x, t)}{\partial t}-D \frac{\partial^{2} c(x, t)}{\partial x^{2}}+\frac{D q \rho(x, t) I(t)}{k_{B} T} \frac{\partial c(x, t)}{\partial x}+ \\
& +\frac{D q I(t)}{k_{B} T} \frac{\partial \rho(x, t)}{\partial x} c(x, t)=S(x, t)
\end{aligned}
$$

This equation should be solved together with the appropriate initial $\mathrm{c}_{\mathrm{in}}(\mathrm{x})$ and boundary conditions. Considering the polar nature of discussed oxides [23], we have calculated intrinsic space charge profiles at equilibrium and found the initial state distribution of vacancies $c_{i n}(x)$ shown in Fig. 3, right (the details will be published elsewhere). Notice also that at comparatively high voltages $\mathrm{qV} / \mathrm{k}_{\mathrm{B}} \mathrm{T} \gg 1$ the contribution of the second term in (3) is small and can be neglected. Physically, it means that at such high voltages the drift of oxygen vacancies in the external field is a dominating factor while the effect from the temperature diffusion is negligible. At last, we have two dimensionless parameters $\beta=\mathrm{qDt}_{0} \rho_{0} \mathrm{I}_{0} /\left(\mathrm{dk}_{\mathrm{B}} \mathrm{T}\right)$, where $\mathrm{I}_{0}$ and $t_{0}$ are amplitude and period of the input current, and $\gamma=\tau / \mathrm{t}_{0}$. The best agreement with the experimental data shown in Fig. 4 was obtained for $\beta=0.07$ and $\gamma=10^{5}$. Results of the simulation are shown in Fig. 4 as well.

\section{Conclusions}

Due to the intense demand for a high-density, high-speed, and low-power nonvolatile memory from the semiconductor industry, in recent years the market of such devices has grown much faster than the entire semiconductor market [24] We focused here on the resistance switching phenomenon in oxide-based heterostructures, its physical mechanisms and explained their memristive behavior. The second part of the paper presents our original results concerning complex transition-metal oxides. Our model simulations and their comparison with the experimental data [22] provide new arguments proving that the resistance switching phenomenon in YBCO-based heterostructures (we suppose that in other complex oxides as well) is directly linked to local resistivity modifications affected by changes of the local oxygen stoichiometry in applied electric fields. Recent STM-contact experiments [25] revealed the same bistability mechanism at the sub-micrometer scale.

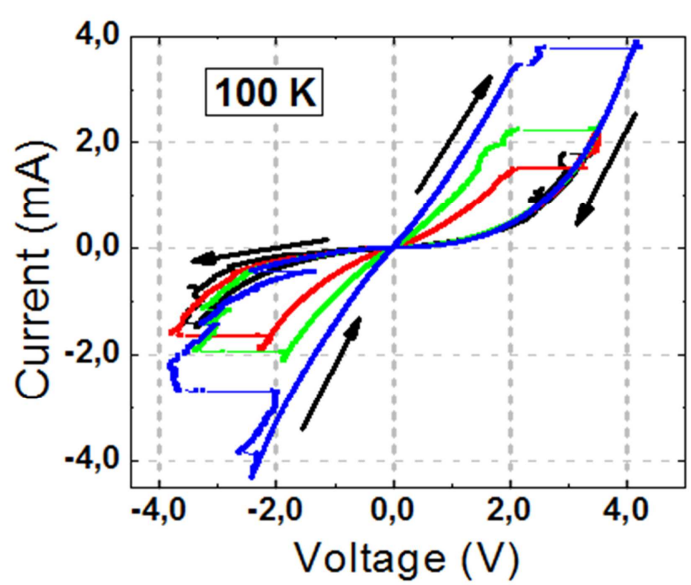

(a)

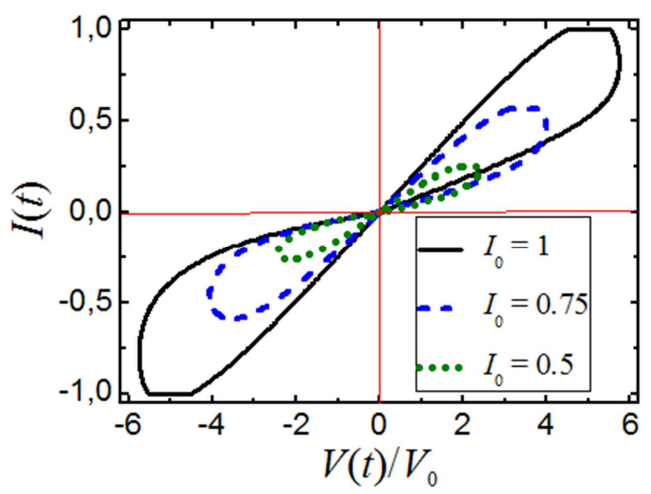

(b)

Figure 4. (a) current-voltage characteristics of the $\mathrm{Ag} / \mathrm{YBCO}$ contact measured at $100 \mathrm{~K}$ which differs by the amplitude $\mathrm{I}_{0}$ of the input alternating current [22]. The bias polarity corresponds to that of the YBCO electric potential. The arrows show direction of $\mathrm{I}(\mathrm{t})$ changes. (b): results of the simulation for the values $\beta=0.07, \gamma=10^{5}$, the input current is a sinusoidal function with the amplitude $\mathrm{I}_{0}, \mathrm{~V}_{0}=\mathrm{I}_{0} \rho_{0} \mathrm{~d}$.

\section{Acknowledgements}

This work has been performed within the grant No. 612600 LIMACONA "Light-Matter Coupling in Composite Nano-Structures" supported by the EU Seventh Framework Programme.

\section{References}

[1] The Machine: A new kind of computer http://www.hpl.hp.com/research/systems-research/themachine/.

[2] L. O. Chua, "Memristor: the missing circuit element", IEEE Trans. on Circuit Theory, vol. 18, pp. 507-519, September 1971.

[3] A. Sawa, "Resistive switching in transition metal oxides", Mater. Today, vol. 11, pp. 28-36, June 2008.

[4] G. Dearnaley, A. M. Stoneham, and D. V. Morgan D V. "Electrical phenomena in amorphous oxide films" Rep. Prog. Phys., vol. 33, pp. 1129-1191, September 1970. 
[5] L. F. Rybalchenko, V. V. Fisun, N. L. Bobrov, I. K. Yanson, A. V. Bondarenko, and A. M. Obolenskii. "Reversible effect of excess current recovery in $\mathrm{Y}(\mathrm{Ho})-\mathrm{Ba}-\mathrm{Cu}-\mathrm{O}$ - normal metal point contacts at high voltages", Fiz. Nizk. Temp., vol. 17, pp. 202-209, February 1991. (in Russian)

[6] L. F. Rybalchenko, N. L. Bobrov, V. V. Fisun, I. K. Yanson, A. G. M. Jansen, and P. Wyder. "Reversible transitions in high- $T_{\mathrm{c}}$ cuprates based point contacts", Eur. Phys. J. B, vol. 10, pp. 475-480, June 1999.

[7] M. A. Belogolovskii, Yu. F. Revenko, A. Yu. Gerasimenko, V. M. Svistunov, E. Hatta, G. Plitnik, V. E. Shaternik, and E. M. Rudenko. "Inelastic electron tunneling across magnetically active interfaces in cuprate and manganite heterostructures modified by electromigration processes", Low Temp. Phys., vol. 28, pp. 391-394, June 2002.

[8] M. A. Belogolovskii. "Interface resistive switching effects in bulk manganites", Cent. Eur. J. Phys., vol. 7, pp. 304-309, June 2009.

[9] D. B. Strukov, G. S. Snider, D. R. Stewart, and R. Stanley Williams. "The missing memristor found", Nature, vol. 453, pp. 80-83, May 2008.

[10] M. J. Rozenberg, I. H. Inoue, and M. J. Sánchez. "Nonvolatile memory with multilevel switching: a basic model", Phys. Rev. Lett., vol. 92, 178302-1 - 178302-4, April 2004.

[11] A. Odagawa, H. Sato, I.H. Inoue, H. Akoh, M. Kawasaki, Y. Tokura, T. Kanno, and H. Adachi, "Colossal electroresistance of a $\mathrm{Pr}_{0.7} \mathrm{Ca}_{0.3} \mathrm{MnO}_{3}$ thin film at room temperature", Phys. Rev. B, vol. 70, pp. 224403-1 - 224403-4, December 2004.

[12] D. S. Shang, Q. Wang, L. D. Chen, R. Dong, X. M. Li, and W. Q. Zhang. "Effect of carrier trapping on the hysteretic currentvoltage characteristics in $\mathrm{Ag} / \mathrm{La}_{0 .}{ }_{7} \mathrm{Ca}_{0.3} \mathrm{MnO}_{3} \quad / \mathrm{Pt}$ heterostructures", vol. 73, pp. 245427-2 - 245427-7, June 2006.

[13] K. Terabe, T. Hasegawa, T. Nakayama, and M. Aono. "Quantized conductance atomic switch", vol. 433, pp. 47-50, January 2005

[14] Y. B. Niam, J. Strozier, N. J. Wu, X. Chen, and A. Ignatiev. "Evidence for an oxygen diffusion model for the electric pulse induced resistance change effect in transition-metal oxides", Phys Rev Lett., vol. 98, pp. 146403-1 - 146403-4, April 2007.

[15] T. Fujii, M. Kawasaki, A. Sawa, Y. Kawazoe, H. Akoh, and Y. Tokura. "Electrical properties and colossal electroresistance of heteroepitaxial $\mathrm{SrRuO}_{3} / \mathrm{SrTi}_{1-x} \mathrm{Nb}_{x} \mathrm{O}_{3} \quad(0.0002 \leq x \leq 0.02)$ Schottky junctions", Phys. Rev. B, vol. 75, pp. 165101-1 165101-7, April 2007.
[16] S. H. Chang, S. C. Chae, S. B. Lee, C. Liu, T. W. Noh, J. S. Lee, B. Kahng, J. H. Jang, M. Y. Kim, D.-W. Kim, and C. U. Jung. "Effects of heat dissipation on unipolar resistance switching in Pt/NiO/Pt capacitors", Appl. Phys. Lett., vol. 92, pp. 183507-1 - 183507-3, May 2008.

[17] D. Lee, S. Baek, M. Ree, and O. Kim. "Unipolar resistive switching characteristic of semiconducting poly (o-anthranilic acid) film", Electronics Lett. vol. 44, pp. 596-597, April 2008.

[18] R. Waser and M. Aono. "Nanoionics-based resistive switching memories", Nat. Mater., vol. 6, pp. 833-840, June 2007.

[19] J. J. Yang, M. D. Pickett, X. Li, D. A. A .Ohlberg, D. R. Stewart, and R. Stanley Williams. "Memristive switching mechanism for metal/oxide/metal nanodevices", Nat. Nanotechnol., vol. 3, pp. 429-433, June 2008.

[20] X. Hu, D. Yang, and J. Hu. "Oxygen diffusion in $\mathrm{YBa}_{2} \mathrm{Cu}_{3} \mathrm{O}_{7-x}$ and its potential applications", in Diffusion and Reactivity in Solids, J. Y. Murdoch, Ed. New York: Nova Science Publ., 2007, pp. 227-241.

[21] K. Yamamoto, B. M. Lairson, J. C. Bravman, and T.H. Geballe. "Oxidation kinetics of $\mathrm{YBa}_{2} \mathrm{Cu}_{3} \mathrm{O}_{7-x}$ thin films in the presence of atomic oxygen and molecular oxygen by in-situ resistivity measurements", J. Appl. Phys., vol. 69, pp. 71897201, June 1991.

[22] T. Plecenik, M. Tomášek, M. Belogolovskii, M. Truchly, M. Gregor, J. Noskovič, M. Zahoran, T. Roch, I. Boylo, M. Španková, Š. Chromik, P. Kúš, and A. Plecenik. "Effect of crystallographic anisotropy on the resistance switching phenomenon in perovskites", J. Appl. Phys., vol. 111, pp. 056106-1 - 056106-3, Match 2012.

[23] H. Su and D. O. Welch. 'The effects of space charge, dopants, and strain fields on surfaces and grain boundaries in YBCO compounds", Supercond. Sci. Tech., vol. 18, pp. 24-34, January 2005.

[24] G. I. Meijer. "Materials science. Who wins the nonvolatile memory race?", Science, vol. 319, pp. 1625-1626, April 2008.

[25] A. Plecenik, M. Tomasek, T. Plecenik, M.Truchly, J. Noskovic, M. Zahoran, T. Roch, M. Belogolovskii, M. Spankova, S. Chromik, and P. Kus. "Studies of resistance switching effects in metal/ $\mathrm{YBa}_{2} \mathrm{Cu}_{3} \mathrm{O}_{7-x}$ interface junctions", Appl. Surf. Sci., vol. 256, pp. 5684-5687, July 2010. 